\title{
Global spiral modes in multi-component disks
}

\author{
N. Orlova ${ }^{1}$, V. Korchagin ${ }^{1}$, and Ch. Theis ${ }^{2}$ \\ 1 Institute of Physics, Stachki 194, Rostov-on-Don, Russia \\ 2 Institut für Theoretische Physik und Astrophysik d. Univ. Kiel, 24098 Kiel, Germany
}

Received 22 June 2001 / Accepted 19 October 2001

\begin{abstract}
We performed two-dimensional non-linear hydrodynamical simulations of two-component gravitating disks aimed at studying stability properties of these systems. In agreement with previous analytical and numerical simulations, we find that the cold gas component strongly affects the growth rates of the unstable global spiral modes in the disk. Already a five percent admixture of cold gas increases approximately two-fold the growth rate of the most unstable global mode while a twenty percent gas admixture enhances the modal growth rate by a factor of four. The local stability properties of a two-component disk coupled by self-gravity are governed by a stability criterion similar to Toomre's $Q$-parameter derived for one-component systems. Using numerical simulations, we analyse the applicability of a two-component local stability criterion for the analysis of the stability properties of global modes. The comparison of non-linear simulations with the analytical stability criterion shows that the two-component disks can be globally unstable while being stable to the local perturbations. The minimum value of the local stability criterion provides, however, a rough estimate of the global stability properties of two-component systems. We find that two-component systems with a content of cold gas of ten percent or less are globally stable if the minimum value of the stability parameter exceeds $\sim 2.5$.
\end{abstract}

Key words. galaxies: kinematics and dynamics - galaxies: spiral

\section{Introduction}

Global spiral arms observed in disk galaxies are the result of density waves propagating in the galactic disks (e.g. Binney \& Tremaine 1987). Starting from pioneering works by B. Lindblad (1941, 1942), a number of authors extensively investigated this idea, and a variety of techniques have been developed to study the stability properties of gravitating disks. Most of these studies concentrated on one-component gaseous or collisionless stellar disks. Galactic disks, however, are multi-component systems whose properties depend on all the constituents. Lin \& Shu (1966) first addressed the question showing the relative importance of gas in providing the spiral gravitational field. Jog \& Solomon (1984) (hereafter JS) considered the influence of a cold component on the stability properties of gravitating disks. They derived a local dispersion relation for a two-fluid instability of a star-gas disk and discussed a relative contribution of two components in the stability properties of the disk. In further investigations the results of JS were generalized by Bertin \& Romeo (1988) who studied the stability properties of the discrete global spiral modes by applying asymptotic WKB-methods. They also

Send offprint requests to: N. Orlova,

e-mail: nata@ip.rsu.ru introduced the concept of the effective $Q$-parameter of the two-component disks. Elmegreen (1995) re-considered the original equations derived by JS and reduced them to a single effective $Q$-parameter. Romeo $(1992,1994)$ included the effects of the finite thickness of stellar and gaseous disks. Recently, Rafikov (2001) gave the analysis of the axisymmetric gravitational stability of the thin rotating disk consisting of several components.

The non-asymptotic global modal stability properties of multi-component disks were studied to a lesser extent. Noh et al. (1991) studied the global stability properties of a thin two-fluid protoplanetary disk composed of gas and dust coupled by gravity and friction and found that the stability properties of these disks are strongly affected by a small amount of dust. Kikuchi et al. (1997) studied global stability properties of a three-phase disk with phases coupled by self-gravity and interchange processes between the phases. Korchagin \& Theis (1999) numerically studied the behavior of global modes in multi-component disks at the non-linear stage of global instability.

In this paper, we return to the problem of the global stability properties of multi-component disks. We consider the simplest possible system of that kind - a two-fluid thin disk coupled by the self-gravity of the components. We compare the results of our non-linear two-dimensional 
numerical simulations with the local stability criteria derived by JS and study the applicability of the Jog-Solomon criterion for the prediction of the global stability properties of multi-phase gravitating disks. We also study the non-linear behaviour of the unstable global modes in multi-phase disks determining the dependence of the saturation levels of the unstable global modes on the mass fraction of a cold component.

Section 2 describes the basic equations and the numerical code used in simulations. In Sect. 3 we present the equilibrium model used in our simulations. In Sect. 4 we briefly describe the two-fluid local stability criterion. Section 5 presents the results of our simulations and gives a comparison of the results of the numerical simulations with the two-fluid analytical stability criterion.

\section{Basic equations}

To model the behaviour of the global perturbations in a gravitationally coupled multi-component disk we use the hydrodynamic approximation, modelling both a stellar and a gaseous component as polytropic fluids:

$P_{\mathrm{s}, \mathrm{g}}=K_{\mathrm{s}, \mathrm{g}} \sigma_{\mathrm{s}, \mathrm{g}}^{\gamma_{\mathrm{s}, \mathrm{g}}}$.

Here $P_{\mathrm{s}, \mathrm{g}}$ are the vertically integrated pressures of each component, $K_{\mathrm{s}, \mathrm{g}}$ are the polytropic constants and $\gamma_{\mathrm{s}, \mathrm{g}}$ are the polytropic indices for stellar and gaseous disks, respectively. In this approach, stellar and gaseous "fluids" differ by the values of their polytropic indices $\gamma_{\mathrm{s}, \mathrm{g}}$. We choose the value $\gamma_{\mathrm{s}}=2$ for the stellar disk which reproduces the empirical "square root" proportionality between the velocity dispersion and the surface density found in galactic disks (Bottema 1992). Additional arguments justifying the description of the collisionless stellar disks by a fluid dynamical approach are given by Kikuchi et al. (1997). The polytropic index for the gaseous disk is assumed to be $\gamma_{\mathrm{g}}=1.66$.

The behaviour of a two-fluid gravitationally interacting system is described by the continuity and momentum equations for each component:

$$
\begin{gathered}
\frac{\partial \sigma_{\mathrm{s}, \mathrm{g}}}{\partial t}+\frac{1}{r} \frac{\partial}{\partial r}\left(r \sigma_{\mathrm{s}, \mathrm{g}} u_{\mathrm{s}, \mathrm{g}}\right)+\frac{1}{r} \frac{\partial}{\partial \phi}\left(\sigma_{\mathrm{s}, \mathrm{g}} v_{\mathrm{s}, \mathrm{g}}\right)=0 \\
\frac{\partial u_{\mathrm{s}, \mathrm{g}}}{\partial t}+u_{\mathrm{s}, \mathrm{g}} \frac{\partial u_{\mathrm{s}, \mathrm{g}}}{\partial r}+\frac{v_{\mathrm{s}, \mathrm{g}}}{r} \frac{\partial u_{\mathrm{s}, \mathrm{g}}}{\partial \phi}-\frac{v_{\mathrm{s}, \mathrm{g}}^{2}}{r}= \\
-\frac{1}{\sigma_{\mathrm{s}, \mathrm{g}}} \frac{\partial P_{\mathrm{s}, \mathrm{g}}}{\partial r}-\frac{\partial}{\partial r}\left(\Psi+\Psi_{\mathrm{H}}\right) \\
\frac{\partial v_{\mathrm{s}, \mathrm{g}}}{\partial t}+u_{\mathrm{s}, \mathrm{g}} \frac{\partial v_{\mathrm{s}, \mathrm{g}}}{\partial r}+\frac{v_{\mathrm{s}, \mathrm{g}}}{r} \frac{\partial v_{\mathrm{s}, \mathrm{g}}}{\partial \phi}+\frac{v_{\mathrm{s}, \mathrm{g}} u_{\mathrm{s}, \mathrm{g}}}{r}= \\
-\frac{1}{\sigma_{\mathrm{s}, \mathrm{g}} r} \frac{\partial P_{\mathrm{s}, \mathrm{g}}}{\partial \phi}-\frac{1}{r} \frac{\partial}{\partial \phi}\left(\Psi+\Psi_{\mathrm{H}}\right)
\end{gathered}
$$

Additionally, all components are coupled by their gravity, which can be expressed by the Poisson equation

$\Psi(r, \phi)=-\int_{R_{\mathrm{in}}}^{R_{\mathrm{out}}} \int_{0}^{2 \pi} \frac{\left(\sigma_{\mathrm{s}}\left(r^{\prime}, \phi^{\prime}\right)+\sigma_{\mathrm{g}}\left(r^{\prime}, \phi^{\prime}\right)\right) r^{\prime} \mathrm{d} r^{\prime} \mathrm{d} \phi^{\prime}}{\sqrt{r^{2}+r^{\prime 2}-2 r r^{\prime} \cos \left(\phi-\phi^{\prime}\right)}}$.
Here $u_{\mathrm{s}, \mathrm{g}}$ and $v_{\mathrm{s}, \mathrm{g}}$ are the radial and azimuthal velocities of stars and gas within the disk, and $\sigma_{\mathrm{s}, \mathrm{g}}$ is the surface density of each component. The potential $\Psi$ derived from the self-gravity of a two-fluid disk, the explicit contribution $\Psi_{\mathrm{H}}$ from the rigid halo, and the pressure gradient determine the behaviour of the disk. All of the dependent variables are functions of the radial coordinate $r$, the azimuthal angle $\phi$, and the time $t$. The variables and the parameters of the model are expressed in units in which the gravitational constant $G$ is equal to unity, the unit of mass is equal to $10^{10} M_{\odot}$ and the unit of length is $2 \mathrm{kpc}$. This choice determines the velocity unit $V_{\text {unit }}=149.1 \mathrm{~km} \mathrm{~s}^{-1}$ and the time unit $T_{\text {unit }}=1.32 \times 10^{7} \mathrm{yr}$.

To solve the two-component hydrodynamical Eqs. (2)(4) we use the multi-component fluid-dynamical code developed by Korchagin \& Theis (1999). The code is a multiphase realization of the Eulerian ZEUS-2D code (Stone \& Norman 1992) with the Van-Leer advection scheme. The two-fluid hydrodynamical equations are solved using equally spaced azimuthal and logarithmically spaced radial zones. In the numerical simulations discussed here we employed a grid of $256 \times 256$ cells. The Poisson equation is solved by the $2 \mathrm{D}$ Fourier convolution theorem in polar coordinates. We look for the global modes starting from initial random perturbations at the level $10^{-6}$.

\section{Equilibrium model}

The equilibrium disk taken in our simulations is based on the equilibrium properties of real galaxies. Specifically, we applied the model of the spiral galaxy NGC 1566 by Korchagin et al. (2000). The disk of NGC 1566 is observationally well studied. Its rotation curve taken from Bottema (1992) can be approximated by

$v_{0}(r)=\frac{V_{1} r}{\left(r^{2}+R_{1}^{2}\right)^{3 / 4}}+\frac{V_{2} r}{\left(r^{2}+R_{2}^{2}\right)^{3 / 4}}$.

The radial dependence of the vertical velocity dispersion of the stars in the disk of NGC 1566 has an exponential distribution

$c_{z}=c_{z}(r)=c_{z 0} \exp \left(-r / h_{c_{z}}\right)$.

This uniquely determines the radial dependence of the axisymmetric surface density distribution assuming that the disk is self-gravitating in the vertical direction:

$\sigma(r)=c_{z}^{2}(r) / \pi G z_{0}$

Here $G$ is the gravitational constant, and $z_{0}$ is the effective thickness of the disk. According to Eqs. (7) and (8), the radial distribution of the stellar surface density can be expressed by

$\sigma_{\mathrm{s}}(r)=\sigma_{0_{\mathrm{s}}} \exp \left(-r / h_{\sigma}\right)\left[1-\left(r / R_{\text {out }}\right)^{2}\right]^{5}$,

where $h_{\sigma}$ is radial scale length for the stellar surface density distribution. For numerical purposes we use in Eq. (9) a multiplier $\left[1-\left(r / R_{\text {out }}\right)^{2}\right]^{5}$ which results in a vanishing 
Table 1. Parameters of the equilibrium model used in the numerical simulations.

\begin{tabular}{ll}
\hline Inner disk radius $\left(R_{\mathrm{in}}\right), \mathrm{kpc}$ & 0.2 \\
Outer disk radius $\left(R_{\text {out }}\right), \mathrm{kpc}$ & 10.0 \\
Disk thickness $\left(z_{0}\right), \mathrm{kpc}$ & 0.7 \\
Scale length of velocity dispersion $\left(h_{c_{z}}\right), \mathrm{kpc}$ & 2.6 \\
Scale length of surface density $\left(h_{\sigma}\right), \mathrm{kpc}$ & 1.3 \\
Central velocity dispersion $\left(c_{z_{0}}\right), \mathrm{km} \mathrm{s}^{-1}$ & 168 \\
Total mass of the disk $\left(M_{\mathrm{d}}\right), M_{\odot}$ & $1.78 \times 10^{10}$ \\
Rotation curve parameters: & \\
$V_{1}, V_{2}, \mathrm{~km} \mathrm{~s}^{-1}$ & 399,82 \\
$R_{1}, R_{2}, \mathrm{kpc}$ & $4.4,0.6$ \\
\hline
\end{tabular}

surface density at the outer boundary of the disk. We further assume that the surface density profile of the gaseous disk and its thickness are the same as those in the stellar disk. However, the total mass of the gaseous disk is smaller compared to the mass of the stellar disk and varies in our models.

The radial velocity dispersion of the stellar and gaseous components can be derived from the equation of state by

$c_{r_{\mathrm{s}, \mathrm{g}}}=\left(\gamma_{\mathrm{s}, \mathrm{g}} K_{\mathrm{s}, \mathrm{g}} \sigma_{\mathrm{s}, \mathrm{g}}(r)^{\gamma_{\mathrm{s}, \mathrm{g}}-1}\right)^{1 / 2}$.

The free parameters $K_{\mathrm{s}, \mathrm{g}}$ can be fixed by the Eqs. (7) and (8) and by the assumption of a constant ratio of the vertical and radial velocity dispersions. For the latter, to get the disk with weak global modes we have chosen $c_{\mathrm{s} z} / c_{\mathrm{s} r}=0.9$. Our choice can be justified as follows. According to Bottema (1992), the measured velocity dispersion in the disk of NGC 1566 gives the real dispersion perpendicular to the galactic disk. In our one-component model, we fix the $z$-component of the velocity dispersion and hence the surface density and the total mass of the disk, and allow for a variation of the radial velocity dispersion. Increasing the ratio $c_{\mathrm{s} z} / c_{\mathrm{s} r}$ we decrease the radial velocity dispersion, hence destabilizing the disk. Simulations show that for the measured $z$-dispersion and rotation curve in the disk of NGC 1566, the disk is stable for the usually assumed ratio $c_{\mathrm{s} z} / c_{\mathrm{s} r}=0.6$. Therefore, we choose in our studies the ratio $c_{\mathrm{s} z} / c_{\mathrm{s} r}=0.9$ which leads to a weakly unstable disk. This ratio is larger than the measured ratio of the velocity dispersions $c_{\mathrm{s} z} / c_{\mathrm{s} r}=0.5-0.6$ in solar neighborhood, and close to the upper limit measured in other galaxies. E.g., the determination of this ratio in the disk of galaxy NGC 488 undertaken by Gerssen et al. (1997) gives the value $0.7 \pm 0.19$. With our choice, however, we do not aim to model any particular galaxy.

The equilibrium rotation of the disk given by the Eq. (6) is balanced by the self-gravity, the pressure gradient of the disk and the external potential of a rigid halo:

$\frac{v_{0}^{2}}{r}=\frac{1}{\sigma} \frac{\mathrm{d} P_{\mathrm{s}}}{\mathrm{d} r}+\frac{\mathrm{d}}{\mathrm{d} r}\left(\Psi+\Psi_{\mathrm{H}}\right)$

By this, we can fix the halo properties. Knowing the initial surface density of the disk we get the disk's self-gravity by solving the Poisson equation numerically. Equation (11) can then be used to calculate the gradient of the halo potential and the halo mass distribution. The general parameters of our equilibrium model are listed in Table 1.

\section{Stability criteria}

The local stability of a thin gravitating disk can be expressed in terms of Toomre's $Q$ parameter which for the gaseous disk has the form (Safronov 1960; Toomre 1964):

$Q \equiv \frac{c(r) \kappa(r)}{\pi G \sigma(r)}$.

Here, $c(r)$ is the sound speed of the gas, $\kappa(r)$ is the epicyclic frequency and $\sigma(r)$ is the surface density of the disk. A gravitating disk is locally unstable when $Q<1$.

The stability criterion (12) was derived for tightly wound perturbations. However, it can be successfully used as an indicator for the stability of global modes in gravitating disks. Numerical simulations show that a locally stable stellar or gaseous disk can be globally unstable if the minimum value of $Q$ does not exceed $\sim 2$. For example, a one-component disk with the rotation and surface density distribution given by Eqs. (6) and (9) can be globally unstable, if the minimum value of $Q$ does not exceed $\sim 1.6$ (Korchagin et al. 2000).

Disks of real galaxies consist of stellar and gaseous components. The stellar component comprises the largest fraction of the total mass of the disk. However, colder gaseous components can play a significant role for the stability properties of multi-component disks. JS derived a local stability criterion for a two-component fluid disk which can be written in the form $Q_{\mathrm{JS}}<1$ where

$Q_{\mathrm{JS}}=\left(\frac{2}{Q_{\mathrm{s}}} \frac{q}{1+q^{2}}+\frac{2}{Q_{\mathrm{g}}} \frac{c_{\mathrm{g}}}{c_{\mathrm{s}}} \frac{q}{1+q^{2} c_{\mathrm{g}}^{2} / c_{\mathrm{s}}^{2}}\right)^{-1}$.

Here $Q_{\mathrm{s}}$ and $Q_{\mathrm{g}}$ are Toomre's $Q$-parameters determined for the "stellar" and gaseous fluids, correspondingly. $q$ is the dimensionless radial wavenumber of perturbations $q=k c_{\mathrm{s}} / \kappa$. The relative contribution $\Gamma$ of gas to stars is given by the ratio of the second to the first term of the stability parameter (13):

$\Gamma=\frac{\sigma_{\mathrm{g}}}{\sigma_{\mathrm{s}}} \frac{1+q^{2}}{1+q^{2} c_{\mathrm{g}}^{2} / c_{\mathrm{s}}^{2}}$.

It can be seen from Eq. (13) that the relative contribution of the components is always larger than the ratio of the surface densities of the components, provided the gas is dynamically colder than the stars. In the limiting case of large wavenumbers $q \rightarrow \infty$ the contribution of the components is given by the ratio $\sigma_{\mathrm{g}} c_{\mathrm{s}}^{2} / \sigma_{\mathrm{s}} c_{\mathrm{g}}^{2}-$ the result, obtained by Marochnik \& Suchkov (1974).

We will use the criterion given by Eq. (13) for the analysis of instability of the global modes in two-component gravitating disks.

\section{Results}

To study the influence of a cold component on the overall dynamics of a two-fluid gravitating disk, we start our 


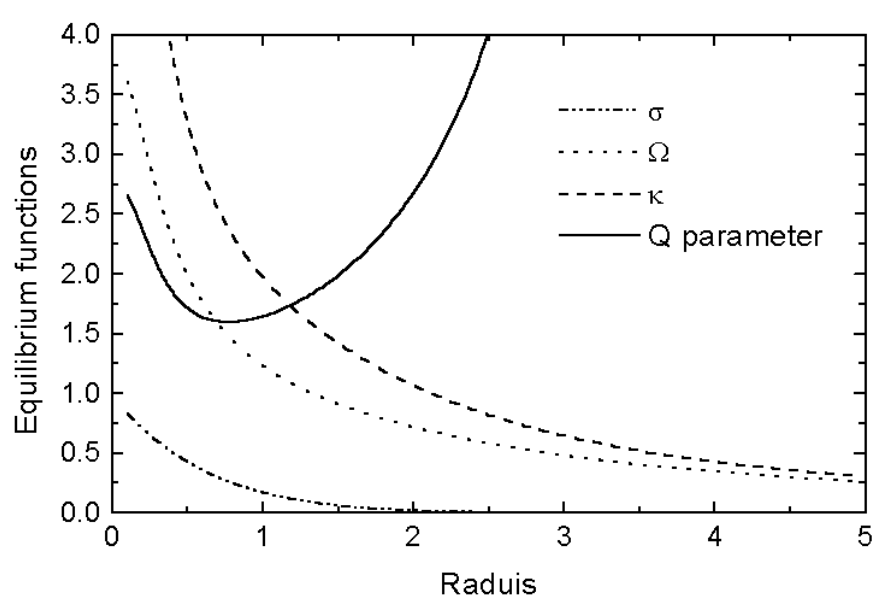

Fig. 1. Equilibrium curves for the one-component "stellar" disk. Angular velocity $\Omega$ and epicyclic frequency $\kappa$ are given in units of $0.75 \times 10^{-7} \mathrm{yr}^{-1}$. The surface density $\sigma$ is given in units $2.5 \times 10^{9} M_{\odot} \mathrm{kpc}^{-2}$, radius is in units of length $L=2 \mathrm{kpc}$.

analysis with a one-component "stellar" disk which we choose to be close to marginal stability. Figure 1 shows the equilibrium properties of this disk. The local stability parameter $Q$ is well above unity with a minimum value $Q_{\min } \sim 1.6$.

The radial behavior of the $Q$-parameter is one of the key assumptions in the modal analyses of spiral density waves. In previous studies it was usually assumed that the profile of the stability parameter $Q$ has a barrier at the central regions of the disk and is equal to unity in the outer regions of the disk (e.g. Bertin et al. 1989). Such a behavior of an "effective" $Q$-parameter is supposed to model the absence of cold gas as well as the transition in geometry from the disk to the nuclear bulge in the central regions of a galaxy, and to describe the increasing role of a cold gaseous component in the outer regions of a galactic disk. In this paper we do not introduce an effective $Q$-parameter, but we try to base our studies on observational properties of disk galaxies. We choose the basic state in our fiducial model based on the properties of the nearby late type spiral galaxy NGC 1566. The "stellar" $Q$-parameter shown in Fig. 1 has an outer barrier which is typical for disks with exponentially decreasing radial surface density distributions and exponentially decreasing velocity dispersions.

Linear global modal analysis of this model reveals a few slowly growing modes with the fastest growing spiral $m=2$. Nonlinear $2 \mathrm{D}$ simulations confirm this result giving an exponential growth rate of the $m=2$ mode of approximately 0.11 in dimensionless units. Linear theory predicts a value of 0.12 which is in good agreement with non-linear simulations. Figure 2 illustrates the evolution of the $m=1-4$ global modes in the one-component "stellar" disk given in terms of the global Fourier amplitudes

$A_{m} \equiv \frac{1}{M_{\mathrm{d}}}\left|\int_{0}^{2 \pi} \int_{R_{\mathrm{in}}}^{R_{\mathrm{out}}} \sigma(r, \phi) r \mathrm{~d} r \mathrm{e}^{-i m \phi} \mathrm{d} \phi\right|$.

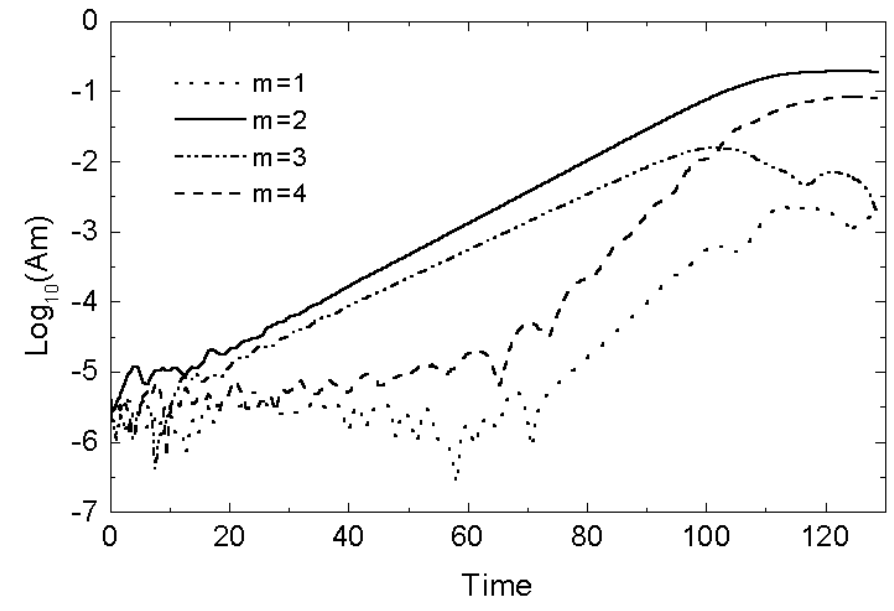

Fig. 2. Temporal evolution of the $m=1-4$ global Fourier amplitudes computed for the one-component "stellar" disk of Fig. 1. Time is in units of $1.32 \times 10^{7} \mathrm{yr}$.

The most unstable $m=2$ mode grows linearly until $t \approx 110$, and then saturates at a level $A_{2} \approx 0.2$ (Fig. 2 ). The linear regime corresponds to an exponential growth of perturbations.

In the next step we split the one-component disk into a "stellar" and a "gaseous" subsystem keeping the total mass of the disk constant. We consider a set of models with a cold component consisting of $5 \%, 10 \%$ and $20 \%$ of the total mass of the disk. In the first series of simulations we keep the "stellar" and gaseous equations of state the same for all the models. As a result, the gas velocity dispersion, which depends on the gas fraction, is not constant. Thus, the velocity dispersion of gas is about $20 \%$ of the velocity dispersion of the stars in the model with $5 \%$ gas admixture. This ratio increases up to $36 \%$ for the model with $20 \%$ gas admixture.

The strong influence of the gas fraction is shown in Fig. 3 which displays the growth rate of the most unstable $m=2$ mode in a one-component "stellar" disk compared to the modal growth rate of the two-component models with $5 \%, 10 \%$, and $20 \%$ gas admixtures. Already a five percent gas fraction enhances the modal growth rate by a factor of 1.7 compared to the one-component disk. A $20 \%$ gas admixture results in an increase of the growth rate by a factor of 3.7. Table 2 gives the masses and the dispersion ratio of the components in the computed models together with the growth rates of the most unstable modes, saturation levels and the minimum values of the two-component stability criterion $Q_{\text {JS }}$ calculated by Eq. (13). The growth rate of the dominant mode increases in the models with higher gas content, being the same for the gaseous and the stellar components. However, the saturation level of the most unstable mode is systematically higher for the gaseous disk. In general, the saturation level slightly decreases in the models with higher gas content. A possible explanation of this behavior is that spiral shocks play a more important role in the saturation of exponentially growing modes when more cold gas is present. 


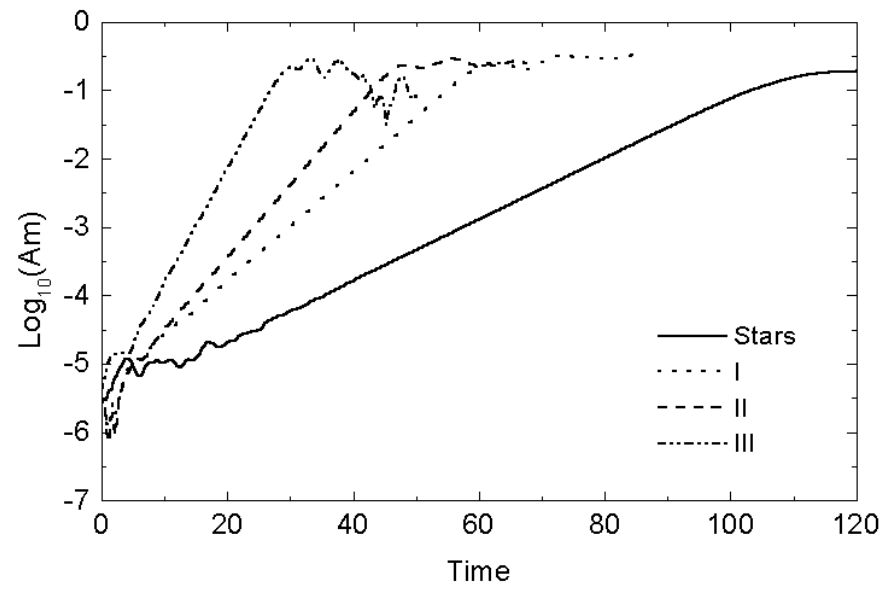

Fig. 3. Time dependence of the global Fourier amplitudes for the most unstable $m=2$ spiral mode computed for models with different gas fractions (I: $5 \%$, II: 10\%, III: 20\%). Time is in units of $1.32 \times 10^{7} \mathrm{yr}$.

Table 2.

\begin{tabular}{c|cccccc}
\hline & & \multicolumn{5}{c}{ Saturation level } \\
Model & $M_{\mathrm{g}} / M_{\mathrm{s}}$ & $c_{\mathrm{g}} / c_{\mathrm{s}}$ & $\mathrm{Im}_{2}$ & gas & stars & $Q_{\mathrm{JS}_{\text {min }}}$ \\
\hline I & 0.05 & 0.2 & 0.18 & 0.35 & 0.27 & 2.2 \\
II & 0.1 & 0.25 & 0.24 & 0.32 & 0.25 & 1.75 \\
III & 0.2 & 0.36 & 0.4 & 0.29 & 0.22 & 1.36 \\
\hline
\end{tabular}

Note. Dependence of the growth rates and the saturation levels on the content of a cold component for the governing $m=2$ mode. The total mass of the disk is fixed to $1.78 \times 10^{10} M_{\odot}$. The growth rates are given in units of $0.75 \times 10^{-7} \mathrm{yr}^{-1}$.

The ratio of the velocity dispersions of the subsystems can also affect the stability of the disk. The influence of the velocity dispersions on the growth rates of the global modes was studied in a series of numerical experiments in which the fraction of the cold component was fixed to $10 \%$ of the total mass of the disk, while the velocity dispersion ratio was allowed to change. The results of these simulations are shown in Fig. 4. The growth rate of the most unstable global mode is only slightly affected by the ratio of the velocity dispersions. The saturation amplitude remains approximately at the same level. Therefore, the mass fraction of the cold component is a more important parameter for the growth rate of perturbations than the ratio of the velocity dispersions. This is in agreement with results of Bertin \& Romeo (1988). They demonstrated (see their Fig. 5) that the criterion of marginal stability of a two-component disk also depends slowly on the ratio of the velocity dispersions of the components and more strongly on the ratio of their densities.

Disks with gaseous admixture show qualitatively the same evolution as the pure stellar disk. However, they evolve on a shorter time scale. The appearance of the global modes in all models is quite similar despite the considerable difference in their growth rates. Figure 5 shows the logarithmically spaced surface density contour plots

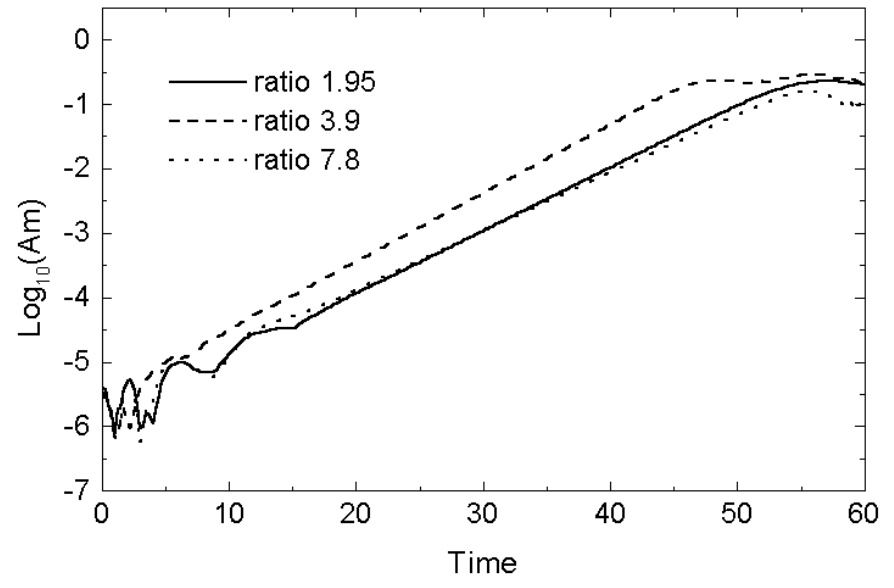

Fig. 4. Time evolution of the global Fourier amplitude for the most unstable mode $(m=2)$ computed for models with $10 \%$ gas admixture and a ratio $c_{\mathrm{rs}} / c_{\mathrm{rg}}$ of the velocity dispersions equal to $1.95,3.9$ and 7.8. Time is in units of $1.32 \times 10^{7} \mathrm{yr}$.

taken at the moments when the global amplitude of the $m=2$ mode in all four models is $A_{2} \approx 0.06$. All the models develop an open two-armed spiral of comparable winding. Note, however, that the spiral arms are more narrow, more tightly wound and less extended when more gas is present. Analysis of the global modes with help of the local dispersion relation (Bertin \& Romeo 1988) also shows a shift towards tighter spirals with a smaller corotation radius in models with higher gas content.

To use the stability criterion (13), one needs to know the wavenumber of the perturbations. Figure 6 shows the radial dependence of the radial wavenumber for $m=2$ perturbations computed for the models I-III. The wavenumbers were determined numerically by calculating the pitch angles of the spiral arms shown in Fig. 5 at different radii, i.e. we used the relation

$\cot i=\left|\frac{k R}{m}\right|$

To find a pitch angle of the spiral at a particular radius, we determine the line of the maximum amplitudes of the spiral arm and calculate the angle between the tangents to the line of maxima and the corresponding circle.

Figure 7 shows the radial profile of the dimensionless Jog-Solomon stability parameter $Q_{\text {JS }}$ computed with the wavenumbers taken from Fig. 6. Figure 7 further illustrates the destabilizing role of a cold gas component: the minimum value $Q_{\mathrm{JS}}$ of the Jog-Solomon parameter decreases with increasing content of cold gas. The minimum value of the Jog-Solomon parameter $Q_{\mathrm{JS}}$ is about 1.36 for the two-component system with $20 \%$ gas admixture versus a minimum value of 2.2 found for the twocomponent model with $5 \%$ gas fraction. In all three models, the Jog-Solomon stability criterion exceeds unity, and all the models are locally stable. Such a behaviour is quite similar to the behaviour of one-component disks which are 

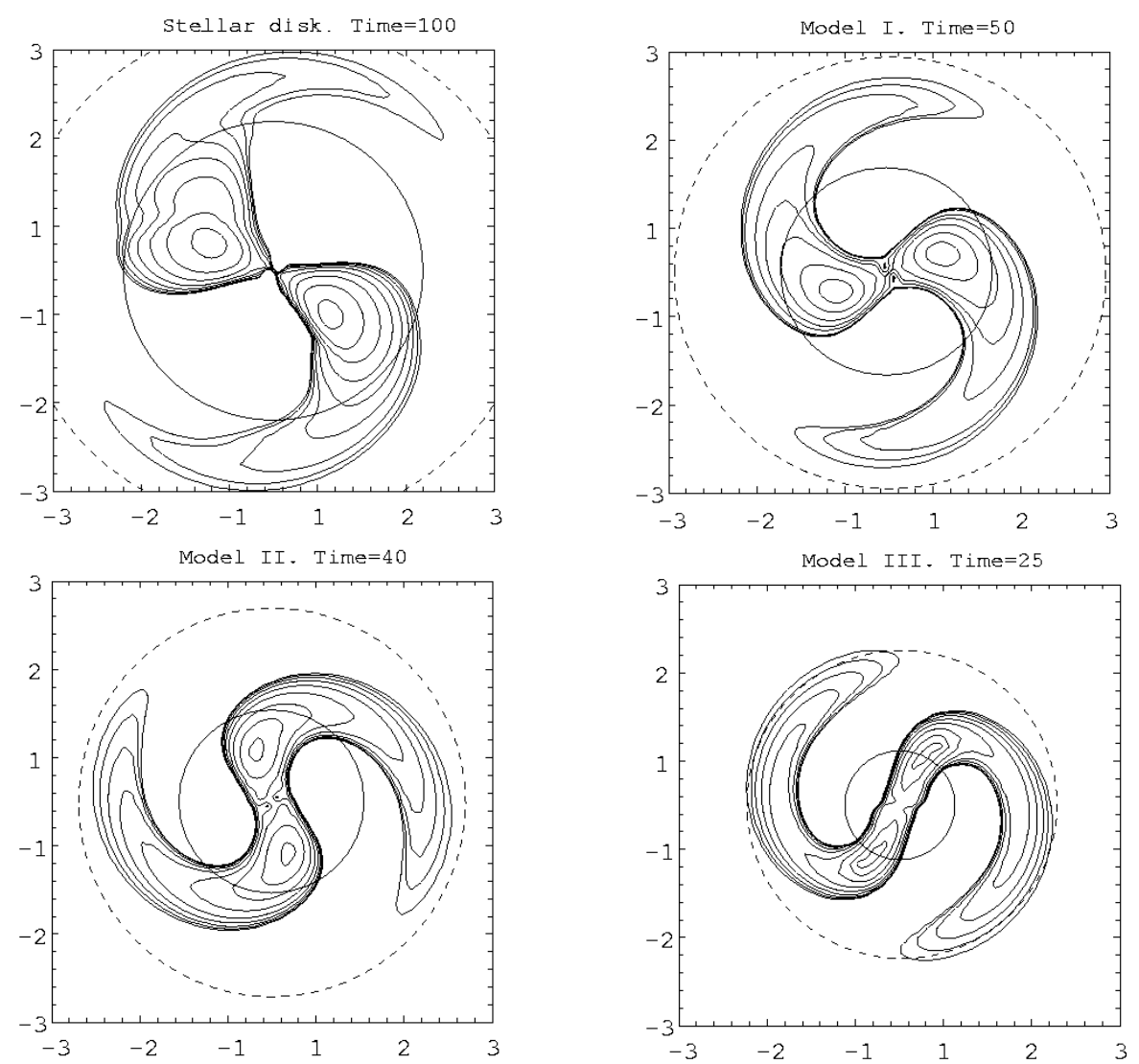

Fig. 5. Surface density perturbations of the stellar component. Displayed are the spiral perturbations in the one-component model (upper left), and in the two-component models with different gas fractions. The snapshots are taken at the moment when the $m=2$ global amplitude reaches $A_{2} \approx 0.06$. The contour levels are logarithmically spaced between the maximum value of the density perturbation and one-hundredth of the maximum value. Circles show the positions of the outer Lindblad resonance (dashed line) and corotation (solid line).

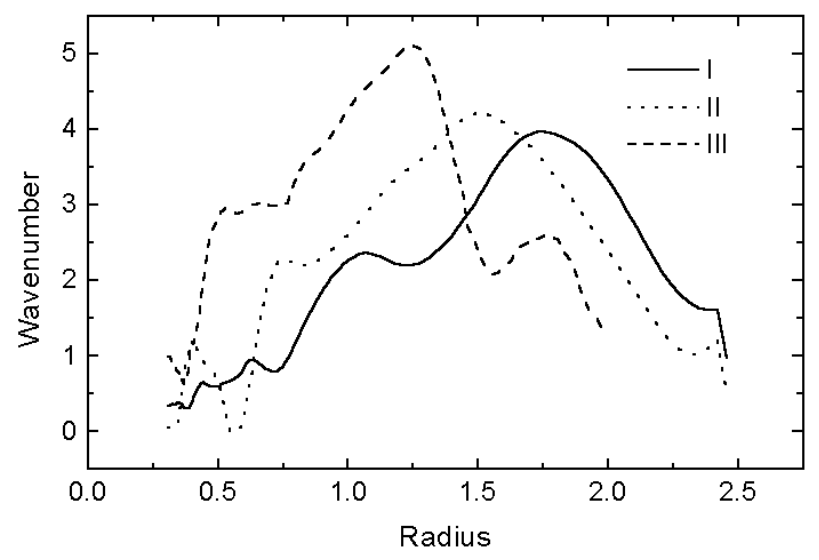

Fig. 6. The radial dependence of the radial wavenumber of two-armed spiral for the models I-III. Unit of length $L=2 \mathrm{kpc}$.

known can be globally unstable even if the minimum value of Toomre's $Q$ parameter is above unity.

Decreasing the total mass of the disk results in its stabilization which consequently leads to an overall growth of the Jog-Solomon parameter $Q_{\text {JS }}$. Figure 8 illustrates this by displaying the growth rate of the dominant mode $m=2$ as a function of the minimum value of the Jog-Solomon

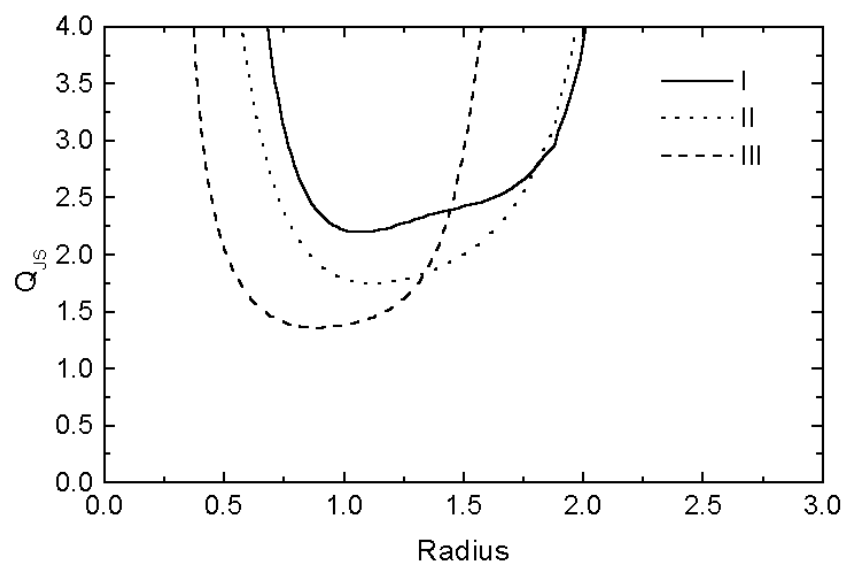

Fig. 7. The radial dependence of the local two-fluid stability parameter $Q_{\mathrm{JS}}$ for disk models with different gas fraction. Unit of length $L=2 \mathrm{kpc}$.

parameter $Q_{\text {JS }}$. The latter was obtained from numerical simulations of two-component disks with $10 \%$ gas admixture. These disks are stabilized when the minimum value of the Jog-Solomon parameter exceeds $\sim 2.5$. 


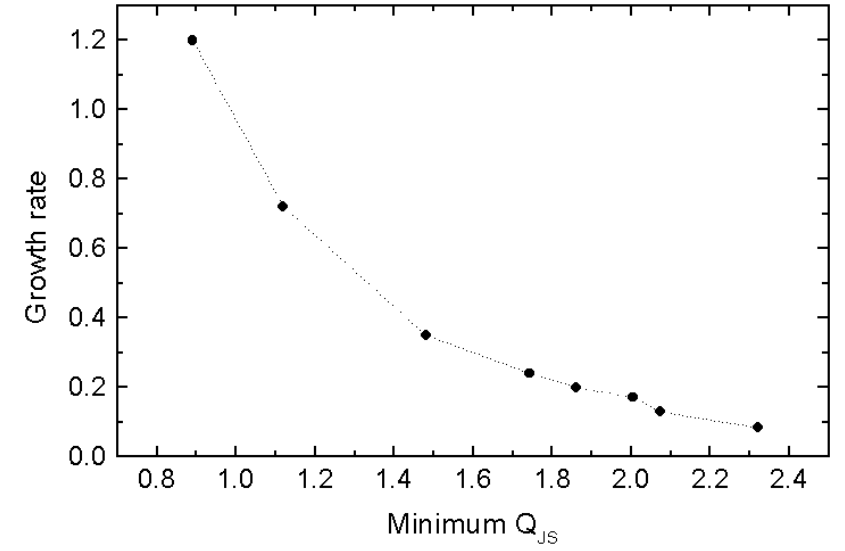

Fig. 8. The growth rate as a function of the minimum value of local stability parameter $Q_{\text {JS }}$.

\section{Summary}

In this paper we studied the applicability of the twofluid local stability criterion derived by Jog \& Solomon (1984) for the onset and growth of non-axisymmetric perturbations in two-component gravitating disks with an open spiral structure. We performed three sets of numerical experiments: one set with fixed total mass of the disk and fixed polytropic equation of state, but variable gas fraction. Another set of simulations was performed with a fixed total mass of the disk and a fixed ratio of the stellar and gaseous velocity dispersions. A final set of calculations deals with a variable disk mass. Our numerical experiments have shown that the mass contribution of the cold component strongly influences the stability properties of the disk. Even a low gas fraction of $5 \%$ destabilizes the disk substantially, e.g. the growth rate of $m=2$ perturbations increases by a factor 1.7. All our disk models are unstable to $m=2$ spiral modes. More unstable disks develop narrower and more tightly wound spiral arms. The saturation levels are almost constant for all simulations, with a slightly enhanced level for the gaseous component. A comparison of the results of the direct two-dimensional numerical simulations with the JS stability criterion allows us to conclude that similarly to Toomre's stability parameter $Q$ derived for one-component disks, the Jog-Solomon stability parameter $Q_{\text {JS }}$ can be used for the prediction of the global stability properties of two-component gravitating disks which greatly simplifies the analysis of disk stability.

Acknowledgements. We thank the referee Dr. A. B. Romeo for his valuable comments on this work. NO and VK gratefully acknowledge support by the Deutsche Forschungsgemeinschaft under grant RUS 17/75/00(S).

\section{References}

Bertin, G., \& Romeo, A. B. 1988, A\&A, 195, 105

Bertin, G., Lin, C. C., Lowe, S. A., \& Thurstans, R. P. 1989, ApJ, 338, 78

Binney, J., \& Tremaine, S. 1987, Galactic Dynamics (Princeton Univ. Press)

Bottema, R. 1992, A\&A, 257, 69

Elmegreen, B. G. 1995, MNRAS, 275, 944

Gerssen, J., Kuijken, K., \& Merrifiel, M. R. 1997, MNRAS, 288,618

Jog, C. J., \& Solomon, P. M. 1984, ApJ, 276, 114

Kikuchi, N., Korchagin, V. I., \& Miyama, S. M. 1997, ApJ, 478, 446

Korchagin, V. I., \& Theis, Ch. 1999, A\&A, 347, 442

Korchagin, V. I., Kikuchi, N., Miyama, S. M., et al. 2000, ApJ, 541,565

Lin, C. C., \& Shu, F. H. 1966, Proc. Nat. Acad. Sci. USA, 55 , 229

Lindblad, B. 1941, Stockholms Obs. Ann., 13, No. 10

Lindblad, B. 1942, Stockholms Obs. Ann., 14, No. 1

Marochnik, L. C., \& Suchkov, A. A. 1974, Uspekhi fiz. Nauk, 112,275

Noh, H., Vishniac, E. T., \& William, D. C. 1991, ApJ, 383, 372

Rafikov, R. R. 2001, MNRAS, 323, 445

Romeo, A. B. 1992, MNRAS, 256, 307

Romeo, A. B. 1994, A\&A, 286, 799

Safronov, V. S. 1960, Ann. Astrophys., 23, 979

Stone, J. M., \& Norman, M. L. 1992, ApJS, 80, 753

Toomre, A. 1964, ApJ, 139, 1217 